\title{
Tuned for the future: Intentions are only accessible when a retrieval opportunity is near
}

\author{
Janette C. Schult • Melanie C. Steffens
}

Published online: 15 June 2013

(C) Psychonomic Society, Inc. 2013

\begin{abstract}
The intention-superiority effect describes faster reaction times for stimuli related to an intention than for more neutral material during the retention interval. Some authors have interpreted the effect in terms of a more persistent activation of intention-related material. However, typically participants are instructed to realize the intention immediately after completing the task in which reaction times are assessed (e.g., a recognition task) in order to yield the intention-superiority effect. Thus, the reaction-time task is also associated with a retrieval context. We tested whether the proximity of a retrieval opportunity affects the intentionsuperiority effect by manipulating whether or not a recognition task indicated a retrieval opportunity. We observed an intention-superiority effect only if the task itself was a meaningful cue for a near retrieval opportunity. This finding indicates that even short-term-delayed intentions flexibly become more or less accessible, depending on the proximity of a retrieval opportunity. We further demonstrated that the intention-superiority effect is not restricted to motor tasks, but is also found for monitoring tasks when the task realization has personally relevant consequences (replicating Schult \& Steffens, Memory \& Cognition, 39, 1487-1495, 2011).
\end{abstract}

Keywords Memory $\cdot$ Intention

J. C. Schult

Social Cognition and Cognitive Psychology, Institute of Psychology, Friedrich Schiller University Jena, Jena, Germany

M. C. Steffens

Social and Economic Psychology, Institute of Psychology,

University of Koblenz-Landau, Landau, Germany

J. C. Schult $(\bowtie)$

Institut für Psychologie, Friedrich-Schiller-Universität Jena,

Am Steiger 3, Haus 1,

D-07743 Jena, Germany

e-mail: janette.schult@uni-jena.de
Prospective memory refers to our ability to retrieve and execute an intended activity at an appropriate moment in the future. A central feature of prospective memory tasks (in contrast to retrospective memory tasks) is the absence of explicit reminders to retrieve the intention when a retrieval opportunity is encountered (e.g., Graf \& Uttl, 2001). For example, if you intend to post a letter, no one tells you to take the letter out of your briefcase when passing a letterbox. Thus, an important question is what brings the representation of an intention into one's mind at an appropriate retrieval opportunity.

One mechanism discussed in the literature is that unfulfilled intentions are represented at a heightened level of accessibility relative to other long-term memory contents (e.g., Ellis, 1996; Förster, Liberman, \& Higgins, 2005; Goschke \& Kuhl, 1993; Lewin, 1926). Heightened accessibility of intention-related concepts could support successful prospective remembering. This heightened accessibility might help with detecting retrieval cues (e.g., Mäntylä, 1996; McDaniel, Guynn, Einstein, \& Breneiser, 2004), or it could lead to more frequent recollections of the intention during the delay interval, which in turn could increase the strength of activation at the present moment and/or initiate monitoring at an appropriate moment to realize the intended activity (e.g., Ellis, 1996). Yet it seems unrealistic that all unfulfilled intentions are represented at a heightened level of accessibility throughout days or weeks (Lebiere \& Lee, 2002; Marsh, Hicks, \& Bink, 1998). Context information indicating the likelihood of a retrieval opportunity in the near future (e.g., finding a letterbox when heading to a shopping tour in the city or a walk in the park) may allow a more flexible accessibility of intentions (e.g., Förster et al., 2005; Marsh, Hicks, \& Cook, 2006). The aim of the present study was to test this proposition.

Previous research using a postponed-intention paradigm has found that stimuli associated with (near-term) intentions were processed faster than equivalent stimuli not associated with intentions during postponement. In this paradigm, 
participants are asked to learn two short lists of actions (e.g., "setting a dinner table" and "clearing a messy desk"). After the study phase, they are instructed to carry out the actions of one list (intention-related list) but not of the other (control) list, after completing a recognition task for both lists. Thus, the realization of the intention has to be postponed until after the recognition task. In the recognition task, reaction times were faster for verbs and nouns of the intention-related list than for stimuli from the control list. Goschke and Kuhl (1993) labeled this reaction-time advantage the intention-superiority effect. The intention-superiority effect has been replicated in a lexicaldecision task (e.g., Dockree \& Ellis, 2001; Marsh et al., 1998; Marsh, Hicks, \& Bryan, 1999; Penningroth, 2011; Schult \& Steffens, 2011) and in more naturalistic settings (Dockree \& Ellis, 2001).

Different mechanisms underlying the intentionsuperiority effect have been discussed. Goschke and Kuhl (1993) interpreted the effect in terms of more persistent activation of intention-related materials. They suggested that intentions are represented as special source nodes that maintain increased levels of subthreshold activation without rehearsal. According to the persistence hypothesis, intention-related concepts ought to be activated for longer periods than are other (long-term memory) concepts after encoding. Alternatively, Freeman and Ellis (2003) proposed that the intention-superiority effect is due to activation of motor or sensorimotor information associated with the future enactment of the postponed task (see Koriat, Ben-Zur, \& Nussbaum, 1990, for a similar reasoning). According to this motor-code hypothesis, an intentionsuperiority effect should be observed for to-be-enacted tasks, but not for tasks without future enactment. Finally, intentions are motivational states. Consequently, factors such as the strength or value of an intention, as well as the perceived likelihood of an opportunity to complete the intention, may affect the intention-superiority effect (Förster et al., 2005). According to this rationale, an intention-superiority effect ought to be more pronounced for important intentions and/or situations in which participants have high expectations of encountering an opportunity to realize the intention. The role of the ongoing (reaction-time) task as a meaningful cue for a likely retrieval opportunity in the near future has not been considered in previous studies using a postponed-intention paradigm.

Specifically, existing studies have demonstrated intentionsuperiority effects immediately after intention formation (Dockree \& Ellis, 2001; Freeman \& Ellis, 2003, Exp. 3; Goschke \& Kuhl, 1993, Exps. 1 and 2; Marsh et al., 1998; Marsh et al., 1999; Penningroth, 2011; Schult \& Steffens, 2011) or after a short filler task (Freeman \& Ellis, 2003, Exp. 4; Goschke \& Kuhl, 1993, Exp. 4). In line with the persistence hypothesis, the results suggest that the accessibility of intentions is increased shortly after encoding and/or decays at a slower rate than other information does. From a practical point of view, it may be functional to keep an intention accessible for such short delays (Marsh et al., 1998). However, in these studies participants have usually been asked to postpone the intention only until after the respective recognition or lexical-decision task that assessed intention superiority (for an exception, see Dockree \& Ellis, 2001). Consequently, the reaction-time task was also associated with a retrieval context: Working on the reaction-time task indicated that an opportunity to complete the intention would come in the near future. The present experiment was designed to disentangle the effects of the persistency of accessibility after encoding and the perceived proximity of a retrieval opportunity on the intention-superiority effect.

Some indirect evidence has suggested that the participants' expectations about the likelihood of a retrieval opportunity affect the accessibility of intention-related information. In prospective memory studies investigating whether holding an intention interferes with performance in an ongoing activity, participants form the intention to press a specific key when encountering a particular event (e.g., an animal word) in an ongoing activity (e.g., a lexical-decision task). The general finding is slower average reaction times in the ongoing activity when holding an intention (i.e., to press a key when animal words are presented) as compared with when no intention is retained. This slowing has been interpreted as a shift in attention allocation away from the ongoing activity and toward the processing of intention-related information. Slower reaction times in an ongoing activity seem particularly likely for activities specified during instructions (e.g., during the second block of the lexical-decision task; see, e.g., Marsh, Hicks, \& Cook, 2008, for a review). This appears to be in line with the possibility that participants' expectations about the likelihood of a retrieval opportunity affect the accessibility of intention-related information. However, the evidence is indirect, based on interference data. Context-sensitive attention allocation should also be reflected in an intention-superiority effect for intentionrelated concepts (for a computational simulation of the intention-superiority effect as a biased attention-allocation policy, see Lebiere \& Lee, 2002).

More direct evidence for context-sensitive accessibility of intentions was reported by Förster et al. (2005). Their participants formed the intention to notify the experimenter when they detected a particular picture sequence. Between blocks of the picture task, participants completed a lexicaldecision task, including stimuli associated with the retrieval opportunity. Förster et al. observed a reaction-time advantage for these intention-related stimuli relative to other stimuli in the lexical-decision task, and the effect was larger in later blocks. Thus, with an increasing likelihood of encountering the intention-related picture sequence, intention- 
related concepts were more accessible. In addition, when they directly manipulated participants' expectations concerning the probability that the critical picture sequence would occur in the experiment, they found a reaction-time advantage for intention-related concepts across all blocks if the occurrence of the critical picture sequence was highly likely, but none if the occurrence of a retrieval opportunity was rather unlikely. Yet this experiment differed in some respects from a postponedintention paradigm, including in its lack of activation of control stimuli in advance. Furthermore, the effect was restricted to concepts related to the retrieval context opportunity (i.e., when to notify the experimenter), whereas intention superiority is usually assessed for content-related information (i.e., what to do during a retrieval opportunity).

To summarize, several experiments on the intentionsuperiority effect have suggested that intention-related concepts enjoy a privileged status in memory in terms of heightened accessibility. However, we know little about the contribution of context information to the accessibility of intentions. Most experiments demonstrating an intentionsuperiority effect have focused on situations in which both the persistent activation of intention-related information and retrieval context information would contribute to the accessibility of intention-related materials. The reaction-time measure was usually associated with a retrieval opportunity during instructions, and the occurrence of the retrieval opportunity in the near future was highly probable. Given previous evidence that retrieval-context information influences the processing of intention-related materials, such context information may be crucial for the occurrence of an intention-superiority effect and may indicate the flexible accessibility of intentions. We tested this possibility below.

A secondary aim of the present experiment was to provide further evidence that intention-superiority effects are independent of the type of postponed task. Freeman and Ellis (2003) proposed that the intention-superiority effect reflects the availability of information associated with future motor execution of the intention, and thus should be restricted to tasks that participants intend to carry out. In line with this reasoning, they reported an intention-superiority effect for verbs that participants intended to enact, but no effect if participants intended to verbally report the verbs. In contrast to a motor-code hypothesis, we provided some evidence that the intentionsuperiority effect may reflect a more general phenomenon of the heightened accessibility of personally relevant intentions, be they related to the future execution of actions or not (Schult \& Steffens, 2011). We found an intentionsuperiority effect in a lexical-decision task for different types of postponed tasks that did not necessarily involve a motor response (e.g., giving a grade for a written recall test), provided that the quality of the task performance was personally relevant for participants. In those experiments, we strengthened personal relevance by announcing a public outcome evaluation: Participants expected to discuss their own performance at the postponed task with a partner (e.g., comparing and justifying their grading decision). We reasoned that public evaluation of one's outcome would strengthen participants' motivation to complete the intention properly (Meacham, 1988). One may object against our findings that intention-related materials were relevant for two successive postponed tasks: for performing the postponed task (e.g., giving a grade) and discussing one's decision in the presence of another participant. Therefore, the present experiment featured only one postponed task; participants either enacted themselves or monitored the experimenter executing the intentionrelated list. To increase participants' motivation to fulfill the postponed task properly, we simply stated that the experimenter would judge participants' performance. Hence, participants expected an outcome evaluation, but they did not have to discuss their performance. If the expectation of an outcome evaluation is a contributing factor in the intention-superiority effect, as we argue, we should replicate the intention-superiority effect for the monitoring intention as well as for the enactment intention in a recognition task.

\section{The present experiment}

Our main aim was to test whether the intention-superiority effect reflects more persistent accessibility of intentionrelated information after encoding, or whether it depends on the association of a reaction-time task with a relevant retrieval context. For all participants, reaction times for intention-related and control materials were assessed in a recognition task. A filler task intervened between the instructions, specifying the intention-related list and the control list, and the beginning of the recognition task. We assumed that a filler task of unspecified length that was unrelated to the recognition task or the intention would prevent participants from keeping the intention accessible in working memory during the whole retention interval. If we were still to demonstrate an intentionsuperiority effect, this would more likely be due to longer-lasting persistent activation or context-sensitive accessibility of the intention-related materials than to active maintaining processes. Goschke and Kuhl (1993) pointed out that intention-related concepts may not constantly remain at a heightened level of activation, but may simply decay at a slower rate than control materials. We therefore used a rather short filler task, assuming that persistent activation should last for more than $1 \mathrm{~min}$ after encoding (cf. Goschke \& Kuhl, 1993, Exp. 4). 
The crucial manipulation was whether or not the recognition task was associated with a proximate retrieval opportunity. Analogously to previous experiments, half of the participants expected to perform the postponed task after the recognition task, which thus indicated a proximate retrieval opportunity. The other half were told to perform the postponed task after the recognition task and another task. In this condition, the recognition task was not associated with a proximate retrieval opportunity, but indicated a more distant retrieval opportunity. If the accessibility of intention-related materials is sensitive to context information, we should observe an intention-superiority effect only when the recognition task was associated with the opportunity to realize the postponed task in the near future.

We also manipulated the type of postponed task between participants. Half of them intended to carry out a list of actions in the presence of the experimenter (enactment task), and the other half intended to monitor and register mistakes in the experimenter's enactment performance (monitoring task). All participants were told that the experimenter would evaluate their performance in the respective task. In line with our previous findings, we predicted that we would observe an intention-superiority effect independent of the type of task (enactment or monitoring) because participants expected to face an evaluation of their outcome (Schult \& Steffens, 2011).

\section{Method}

\section{Participants}

A group of 49 undergraduates (age range 18-39 years, $M=22, S D=3.8 ; 81 \%$ women) volunteered in exchange for course credit. One participant (proximate retrieval opportunity, monitoring task) who thought that the experiment was finished after the recognition task was replaced. Twelve students were randomly assigned to each condition.

\section{Materials}

The study materials were modeled after those of Goschke and Kuhl (1993). Two lists describing simple activities (brewing coffee or making a sandwich) were used. Each list consisted of six verb-object phrases. The stimuli of both study lists were comparable with regard to word length $[M=$ 7.4 for brewing coffee and $M=7.5$ for making a sandwich; $t(22)<1]$ and word frequency (frequency class: $M=14.5$ for both lists, according to the Deutscher Wortschatz, 2013). The recognition task consisted of 24 list words (the six verbs and six nouns of each study list) and 24 distractor words (eight lure words related to brewing coffee, eight lure words related to making a sandwich, and eight unrelated words). ${ }^{1}$

\section{Procedure}

The participants were tested individually. When entering the laboratory, they were seated in front of a computer. The experimenter left the room with the remark: "If you need me, I am in the room next door." All instructions were presented on a computer screen. At the beginning, participants were instructed to learn two lists of actions for a later (unspecified) memory task, emphasizing that they should be able to distinguish what action belonged to which list. The presentation of the study materials followed Goschke and Kuhl's (1993) procedure: First, a list title (e.g., "List 1") and then each action of a list appeared on the screen, one at a time for $5 \mathrm{~s}$. Next, the whole list (and the list title) was presented for $30 \mathrm{~s}$. Then the second list was presented in the same way. Both lists were presented alternately three times. The first-presented list was always called "List 1" and the second one "List 2." The overall topics of the lists (e.g., brewing coffee) were not mentioned. The presentation order of the two lists was counterbalanced across participants, and each list served equally often as List 1 and List 2.

After studying both lists, participants were informed that they were going to work on a series of tasks, and each task was explained briefly. First, the filler task was explained as "write down as many different examples of a given category within $30 \mathrm{~s}$ as possible," and an example ("flowers, ... rose, aster, geranium") was given. Participants expected to complete this generation task for an unspecified number of

\footnotetext{
${ }^{1}$ We were also interested in whether intentions are represented in a specific or a more general form (e.g., brewing coffee after the memory test). Therefore, we included lure words related to both lists in the recognition task. We assumed that if participants formed a more general intention, this should be reflected in slower reaction times and higher false alarm rates when rejecting intention-related lures as compared to lures related to the control list or new words. A 3 (word type: intention-related, control, new) $\times 2$ (retrieval opportunity) $\times 2$ (performance task) ANOVA for rejection times revealed a main effect of word type, $F(2,88)=28.81, R_{\mathrm{p}}^{2}=.40$. Planned contrasts showed that rejection times did not differ between the different types of lure words ( $M=1,059 \mathrm{~ms}$ for the intention-related list and $M=1,064 \mathrm{~ms}$ for the control list; $F<1$ ), and lure words were rejected more slowly than new distractor words $(M=908 \mathrm{~ms}), F(1,44)=48.67, R_{\mathrm{p}}^{2}=.53$. Similarly, we observed a main effect of word type for false alarm rates, $F(2,88)=$ $16.35, R_{\mathrm{p}}^{2}=.27$. The false alarm rates for lure words related to the intention-related list $(M=.13)$ and the control list $(M=.12)$ were comparable $(F<1)$, and false alarm rates were higher for lure words than for new distractor words $(M=.03), F(1,44)=46.44, R_{\mathrm{p}}^{2}=.51$. Thus, in the present experiment it seems that the heightened accessibility of intention-related materials was specific to the learned actions. However, stimulus encoding with arbitrary list titles (e.g., "List 1") instead of a general topic (e.g., "brewing coffee") might have prevented a more generalized representation of the to-be-realized task.
} 
categories. Second, the recognition task was explained. Participants were told that a word would be presented in the middle of the screen. They should press the " $y$ " key as fast as possible if the word had appeared in one of the two studied lists, or the " $n$ " key if it had not. Both keys were marked with yellow dots. Third, a performance task was announced. Half of the participants were told that they were going to carry out the actions of one list in a nearby kitchen in the presence of the experimenter (enactment task). The other half of participants were told that they were going to monitor the experimenter's execution of one list of actions and that they should notice all errors (monitoring task). To make sure that participants perceived their respective tasks as being personally relevant, it was stated that the experimenter would evaluate their performance. An incomplete execution of the list of actions or the nondetection of errors in the experimenter's performance would lead to a poor evaluation. Thus, the monitoring task was very similar to previous attempts that had yielded no reaction-time differences between intention-related and control materials (Goschke \& Kuhl, 1993; Marsh et al., 1998). The major difference was that the participants in our monitoring condition were asked to produce an objective performance outcome (which errors did the experimenter make?) that would be evaluated by the experimenter.

In addition to the type of performance task, we manipulated participants' expectations about when to realize the task. Half of the participants were instructed to notify the experimenter that they were ready to complete the performance task as soon as they had finished the memory task - that is, the recognition task (proximate retrieval opportunity). The other half of the participants was asked to notify the experimenter after they had finished the pretest for another experiment following the recognition task. The pretest was for participants to generate typical characteristics of a particular student group. Although these participants worked on the recognition task at the same time as participants of the other group had (after the generation task), the recognition task was not relevant as a retrieval opportunity (distant retrieval opportunity). After the task explanations, the words "after the memory task" (proximate retrieval opportunity) or "after the pretest" (distant retrieval opportunity) were displayed. After $2 \mathrm{~s}$, the computer beeped, and information specifying which list had to be executed or monitored, respectively, in the performance task was presented for $3 \mathrm{~s}$ : One sentence indicated the intentionrelated list (e.g., "enact List 1"), the other one indicated the control list (e.g., "do not enact List 2"). Both study lists served equally often as the intention-related and the control list.

Subsequently, participants completed the explained tasks. Each task (generation task, memory task, or pretest) was announced for $3 \mathrm{~s}$ before the first trial of the respective task started. The generation task consisted of two trials, each lasting $30 \mathrm{~s}$. Different categories (e.g., furniture) were used across participants, and between the trials participants could rest for $5 \mathrm{~s}$. During the break, the information "Continuing soon" was displayed. Thus, the time interval between intention formation and the beginning of the recognition task was about $1 \mathrm{~min}$. Next, the words "memory task" were presented. The related future performance task was not mentioned at that moment. During the recognition task, the words "old" and "new" were displayed at the left and right upper sides of the screen. Each trial started with a fixation cross for $250 \mathrm{~ms}$, followed by the presentation of the stimulus in the middle of the screen. The word remained on the screen until participants pressed the " $\mathrm{y}$ " or " $\mathrm{n}$ " key. If participants pressed the wrong key, they received auditory feedback (a beep). The intertrial interval was $500 \mathrm{~ms}$. In the proximate retrieval condition, the word "End" appeared on the screen after the last trial of the recognition task. In the distant retrieval condition, the word "Pre-test" appeared on the screen, and participants wrote down typical characteristics of a particular student group. The test ended after $30 \mathrm{~s}$, and the word "End" appeared on the screen.

When participants approached the experimenter, they were asked why they had come. Participants expected that they were going to enact or monitor the intention-related list in a nearby kitchen. Instead of performing the enactment or monitoring task, however, participants were seated again in the laboratory and responded to a computer-based questionnaire. They indicated which list they had intended to realize (List 1, List 2, or "don't know") and what title they would give to that particular list ("making a sandwich," "brewing coffee," or "don't know") as a manipulation check. Finally, a free-recall test of the intention-related list was administered.

\section{Design}

Retrieval opportunity (proximate vs. distant) and performance task (enactment vs. monitoring) were manipulated between subjects. List type (intention-related, control) was manipulated within subjects. The main dependent variable was recognition latencies.

\section{Results}

For all statistical analyses, the Type I error was set at $\alpha<.05$.

Preliminary analyses

The total reading times for the instructions did not differ between groups (all $F_{\mathrm{S}}<1.4$ ). All participants indicated the correct intention-related list (List 1 or List 2) and the correct topic (brewing coffee or making a sandwich). All of the participants in the enactment condition completely recalled 
the intention-related list. Three participants in the monitoring condition recalled at least four of the six actions. In sum, free recall was rather good, indicating that the study time was sufficient to learn the materials. The reported pattern of results did not change when participants with imperfect recall were excluded.

As an index of recognition accuracy, we computed the proportion of items correctly classified as "old" for each condition (cf. Freeman \& Ellis, 2003). No significant effects emerged in a 2 (retrieval opportunity) $\times 2$ (performance task) $\times 2$ (list) mixed ANOVA, all $F_{\mathrm{s}}<2$. As is shown in Table 1, recognition accuracy was at ceiling, corroborating that the study time was sufficient.

\section{Main analyses}

Following Goschke and Kuhl (1993), incorrect trials (9 \% of the total number) and reaction times beyond three $S D$ s of each participant's individual mean response $(2 \%)$ were excluded from the analyses, and statistical analyses were carried out on $\log$-transformed scores to arrive at a normal distribution. Table 1 presents the mean reaction times for the studied stimuli of both lists. Reaction times for the correctly recognized stimuli from the intention-related and control list were analyzed in a 2 (retrieval context) $\times 2$ (performance task) $\times 2$ (list) mixed ANOVA. In line with the results of a previous study (Schult \& Steffens, 2011), no effects involving the factor Performance Task were significant, all $F_{\mathrm{s}}<1.3$. We did find a main effect of list, $F(1,44)=4.64, R_{\mathrm{p}}^{2}=.10$, and this effect was qualified by a List $\times$ Retrieval Opportunity interaction, $F(1,44)=9.03, R_{\mathrm{p}}^{2}=.17$ (see Fig. 1). In line with the context-sensitive accessibility of intention-related information, an intention-superiority effect was apparent when the recognition task was associated with a proximate retrieval opportunity [simple main effect: $F(1,44)=13.3, R^{2}=.23$ ], and reaction times were comparable for stimuli from the

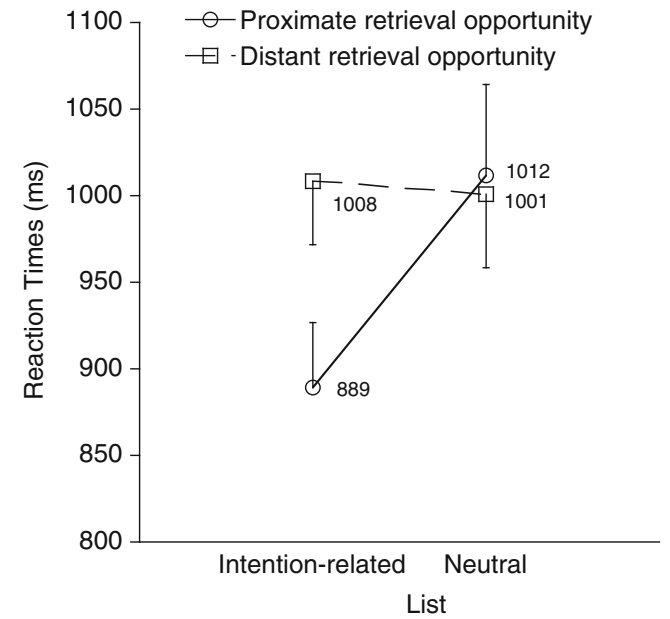

Fig. 1 Mean response latencies in the recognition task, presented separately for the proximate retrieval opportunity and the distant retrieval opportunity conditions and for the intention-related versus control lists. Error bars show standard errors of the means

intention-related and control lists if the recognition task was not a relevant retrieval context (simple main effect: $F<1$ ). Conversely, reaction times for intention-related stimuli were faster if the recognition task was associated with a retrieval opportunity than if it was not [simple main effect: $F(1,44)=$ $13.3, R_{\mathrm{p}}^{2}=.23$ ], whereas reaction times were comparable across conditions for stimuli of the control list $(F<1)$.

\section{Discussion}

The present experiment has demonstrated two interesting findings. First, retrieval context information affected the accessibility of intention-related information in a postponedintention paradigm. We found an intention-superiority effect if the recognition task was associated with an opportunity to

Table 1 Mean reaction times and hit rates (with standard deviations) for stimuli from the intention-related and control lists, presented separately by retrieval opportunity and performance task

\begin{tabular}{|c|c|c|c|c|c|c|c|c|}
\hline \multirow{3}{*}{$\begin{array}{l}\text { Retrieval Opportunity } \\
\text { List } \\
\text { Performance Task }\end{array}$} & \multicolumn{4}{|c|}{ Proximate } & \multicolumn{4}{|c|}{ Distant } \\
\hline & \multicolumn{2}{|c|}{ Intention-Related } & \multicolumn{2}{|c|}{ Control } & \multicolumn{2}{|c|}{ Intention-Related } & \multicolumn{2}{|c|}{ Control } \\
\hline & $M$ & $S D$ & $M$ & $S D$ & $M$ & $S D$ & $M$ & $S D$ \\
\hline \multicolumn{9}{|l|}{ Reaction Times } \\
\hline Enactment & 889 & 185 & 974 & 178 & 1,051 & 159 & 1,031 & 197 \\
\hline Monitoring & 889 & 198 & 1,050 & 317 & 965 & 190 & 971 & 225 \\
\hline \multicolumn{9}{|l|}{ Hits } \\
\hline Enactment & .96 & .08 & .89 & .09 & .89 & .10 & .93 & .11 \\
\hline Monitoring & .88 & .14 & .90 & .12 & .92 & .12 & .92 & .08 \\
\hline
\end{tabular}


realize the intention. Although reaction times were assessed at the same moment as in the proximate retrieval condition, no intention-superiority effect emerged if the recognition task signaled no retrieval opportunity in the near future. The latter finding is not easily compatible with the persistence hypothesis: If intentions simply persisted at a higher level of accessibility after intention formation than did other materials, we should have observed an intention-superiority effect in both retrieval conditions. Yet, about $1 \mathrm{~min}$ after intention formation, reaction times were comparable for intention-related and control stimuli in the distant retrieval condition.

The second finding was that intention-related information was processed faster in the proximate retrieval condition if this intention was relevant for the participants, whether or not it was related to a motor response. Descriptively, the intention-superiority effect was even larger for the monitoring condition than for the enactment condition. This extends the previous finding of comparable results for different types of postponed tasks (Schult \& Steffens, 2011) to another type of assessment (recognition instead of a lexicaldecision task) and to a more subtle manipulation of personal relevance. In other words, the announcement of an evaluation from another person seems sufficient to induce a feeling of personal relevance. In sum, the present findings indicate that motivational aspects such as evaluation apprehension, rather than participants' active involvement in an evaluation, contribute to the heightened activation of intentions in a postponed-intention paradigm. Future motor recall is no precondition for the heightened accessibility of intentionrelated concepts, as was proposed by Freeman and Ellis (2003), if the intention is highly relevant (for a more extended discussion, see Schult \& Steffens, 2011). It would be worth while for future research to examine the degree of personal relevance that is needed in order to observe a heightened accessibility of nonmotor intentions.

The present findings fit nicely into the context-switching account of the intention-superiority effect that Lebiere and Lee (2002) proposed using the ACT-R cognitive architecture (e.g., Anderson \& Lebiere, 1998). The main idea behind their modeling studies was that uncompleted intentions provide the context for the present goal, priming the appropriate memory items. "If a task is expected to be performed in the near future (and no other pressing one is currently being performed) then the context is set to that task to facilitate the retrieval of related information" (p. 62). In line with the parenthetical in that quotation, our findings demonstrate that the task preceding the intention needs to be taken into account in the goal-activation process. We suppose that it activates the context. However, as we demonstrated, if it is not associated with the intended task, because an intervening task serves as a pressing task before "the near future," then no extra activation of intention-related stimuli can be observed. Lebiere and Lee had already acknowledged that it would clearly not be possible for prospective tasks to remain accessible until the time to perform them if longer time intervals in a busy schedule were concerned. In relation to the secondary aim of the present research, those authors hypothesized that the context is set only to a to-be-performed task, but not to a "less demanding observe condition" (p. 62). Going beyond that, our findings demonstrate that a monitoring condition (which is similar to an observe condition) can indeed lead to findings equivalent to those from a performance condition as soon as the monitoring condition is not perceived as being less demanding. This can easily be incorporated into their model by assuming that the context is set to any task, performance or monitoring, that appears demanding and important.

Parallels exist between the context-sensitive intentionsuperiority effect in our experiment and the research on task interference undertaken by Marsh and colleagues (Cook, Marsh, \& Hicks, 2005; Marsh et al., 2006, 2008). For example, Marsh et al. (2006) examined task interference from keeping a time-based intention while working on a lexical-decision task. They observed slower responses to neutral stimuli than in a nointention control condition across two blocks of the lexicaldecision task if the retrieval context was ill-defined (i.e., to press a specific key in about $6 \mathrm{~min}$ ). If, however, participants received the additional information that the response window would occur in the second block, task interference effects were restricted to that particular block. Some authors have attributed task interference effects to monitoring processes: To some extent, attentional resources are devoted to the detection of intention-related information during a delay interval (e.g., Guynn, 2003; Smith, 2003, 2008). More recently, Smith (2008) suggested that such monitoring processes could be restricted to intervals in which a retrieval opportunity was likely. In line with this reasoning, we demonstrated an intention-superiority effect under conditions associated with a retrieval opportunity. We demonstrated the context-sensitive accessibility of shortly delayed intentions. By extending these findings, we would expect an intention-superiority effect even after a longer delay, provided that the reaction-time task signaled a retrieval opportunity in the near future.

So far, we have interpreted faster reaction times for intention-related words in a proximate retrieval context in terms of a context-sensitive speeded processing of intentions. An alternative interpretation is a slowing of currently to-be-ignored information (the control list and/or the intention in the distant condition). As Marsh et al. (1998) noted, the intention-superiority effect-that is, the comparison of intention-related and control stimuli-is a relative reactiontime advantage, to which the heightened accessibility of intention-related information, the inhibition of irrelevant information (i.e., the control list), or a combination of both might contribute. In the present experiment, we cannot 
distinguish between these interpretations. A no-intention control condition included in future experiments might be able to resolve this question.

The present experiment shows that cues related to a retrieval context affect the accessibility of intentions. However, we did not disentangle whether an intentionsuperiority effect occurred because the beginning of the recognition task was associated with a retrieval context per se or because participants expected to fulfill the postponed task in the near future. If participants' expectations about the distance of a retrieval opportunity were crucial, one would expect to find an intention-superiority effect in later intervals of a prolonged recognition task, but not at the beginning. Some findings have suggested the flexible accessibility of intentions within an associated retrieval context. Goschke and Kuhl (1993, Exp. 3) instructed their participants to work on a recognition task and to interrupt the task when they felt that $15 \mathrm{~min}$ had elapsed. The recognition task consisted of blocks in which the same learned words, as well as distractor words, were presented repeatedly. Goschke and Kuhl computed reaction times for intention-related and control words in the first, individual center, and final list intervals; they found an intention-superiority effect in the first block of a recognition task, as well as immediately before participants expected to carry out the intention, but not in the center block, in which the intention was irrelevant. This pattern of results was recently replicated (Schult, 2011). In both studies, the intention-superiority effect in the first block seems to contradict our notion of a context-sensitive accessibility of intentions. However, the recognition task always started immediately after participants had been told what to do after the recognition task. No task intervened between the intention formation and the beginning of the retrieval context. Thus, the intention-superiority effects immediately after intention formation might reflect intention-formation processes to some extent, rather than context-sensitive accessibility.

In the present experiment, we cannot rule out the possibility that the demonstrated intention-superiority effect reflects strategic rehearsal processes (Altmann \& Trafton, 2002). The memory task was announced $3 \mathrm{~s}$ before the first recognition trial started. This short period of time may have been sufficient to (partially) rehearse the topic or specific actions of the more important, intention-related list. However, our finding of a context-sensitive intention-superiority effect suggests that such rehearsal strategies during breaks are particularly used when a retrieval context is encountered. If participants rehearsed the intention-related list, this rehearsal seems to have been rather specific. A general rehearsal of the to-be-realized task (e.g., brewing coffee) should have resulted in slower rejection latencies for intention-related distractors relative to distractors associated with the control list. Neither reaction times nor false alarm rates differed for intention-related and control distractor stimuli in the present experiment (see note 1). In addition, other findings - such as an intention-superiority effect for lists of unrelated actions (Marsh et al., 1999), or similar intention-superiority effects for participants who reported biased rehearsal of the intention-related list and for those who reported similar rehearsal of both lists before a recognition task (Goschke \& Kuhl, 1993) — also suggest that the intention-superiority effect is due to more than strategic rehearsal.

Several recent experiments have demonstrated the lingering accessibility of intentions after intention completion (e.g., Penningroth, 2011; Walser, Fischer, \& Goschke, 2012). In contrast, we have shown that accessibility before intention completion depends on the proximity of a perceived retrieval opportunity. Thus, these findings do not contradict each other. For a discussion of the hypothesized processes after intention completion, see Walser and colleagues (2012).

To conclude, the postponed-intention paradigm has so far demonstrated the increased accessibility of short-term delayed intentions across an intervening task. However, a cognitive system that extended this accessibility level across hours, or even longer, would be highly dysfunctional. In line with this notion, as we have shown, even the accessibility of short-term activity-based intentions waxes and wanes, depending on the proximity of a retrieval opportunity. Environmental cues, such as arriving in a context associated with an intention, are used to gain such information. A heightened accessibility of delayed intentions during moments in which a retrieval opportunity is likely to occur increases the chances that the opportunity will be detected, improving the probability of intention completion. Such a flexible accessibility of intentions is highly functional whenever execution has to be considerably postponed.

Author Note The writing of this article was supported by grants from the Deutsche Forschungsgemeinschaft (German Science Foundation; Grant Nos. Ste 938/7-1 and Ste 938/7-2). We thank Sascha Poppitz, Claudia Richter, Linda Seidemann, and Melanie Wollner for support with the data collection. We also thank Micheal Scullin and two anonymous reviewers for their helpful comments on an earlier version of this article.

Please address correspondence to Janette Schult, Institut für Psychologie, Friedrich-Schiller-Universität Jena, Am Steiger 3, Haus 1, D-07743 Jena, Germany, E-mail:janette.schult@uni-jena.de. Phone: +49-3641-945 114. Fax: +49-3641-945 112."

\section{References}

Altmann, E. M., \& Trafton, J. G. (2002). Memory for goals: An activation-based model. Cognitive Science, 26, 39-83. doi:10.1207/s15516709 $\operatorname{cog} 2601 \_2$

Anderson, J. R., \& Lebiere, C. (1998). Atomic components of thought. Mahwah, NJ: Erlbaum. 
Cook, G. I., Marsh, R. L., \& Hicks, J. L. (2005). Associating a timebased prospective memory task with an expected context can improve or impair intention completion. Applied Cognitive Psychology, 19, 345-360. doi:10.1002/acp.1082

Deutscher Wortschatz [Database]. (2013). Retrieved from http:// wortschatz.uni-leipzig.de/index.html

Dockree, P. M., \& Ellis, J. A. (2001). Forming and canceling everyday intentions: Implications for prospective remembering. Memory \& Cognition, 29, 1139-1145. doi:10.3758/BF03206383

Ellis, J. (1996). Prospective memory or the realization of delayed intentions: A conceptual framework for research. In $M$. Brandimonte, G. O. Einstein, \& M. A. McDaniel (Eds.), Prospective memory: Theory and applications (pp. 1-22). Mahwah, NJ: Erlbaum.

Förster, J., Liberman, N., \& Higgins, E. T. (2005). Accessibility from active and fulfilled goals. Journal of Experimental Social Psychology, 41, 220-239. doi:10.1016/j.jesp.2004.06.009

Freeman, J. E., \& Ellis, J. A. (2003). The representation of delayed intentions: A prospective subject-performed task? Journal of Experimental Psychology: Learning, Memory, and Cognition, 29, 976-992. doi:10.1037/0278-7393.29.5.976

Goschke, T., \& Kuhl, J. (1993). Representation of intentions: Persisting activation in memory. Journal of Experimental Psychology: Learning, Memory, and Cognition, 19, 1211-1226. doi:10.1037/0278-7393.19.5.1211

Graf, P., \& Uttl, B. (2001). Prospective memory: A new focus for research. Consciousness and Cognition, 10, 437-450. doi:10.1006/ccog.2001.0504

Guynn, M. J. (2003). A two-process model of strategic monitoring in event-based prospective memory: Activation/retrieval mode and checking. International Journal of Psychology, 38, 245-256. doi:10.1080/00207590344000178

Koriat, A., Ben-Zur, H., \& Nussbaum, A. (1990). Encoding information for future action: Memory for to-be-performed tasks versus memory for to-be-recalled tasks. Memory \& Cognition, 18, 568578. doi:10.3758/BF03197099

Lebiere, C., \& Lee, F. J. (2002). Intention superiority effect: A contextswitching account. Cognitive Systems Research, 3, 57-65. doi:10.1016/S1389-0417(01)00044-4

Lewin, K. (1926). Vorsatz, Wille und Bedürfnis. Psychological Research, 7, 330-385. doi:10.1007/BF02424365

Mäntylä, T. (1996). Activating actions and interrupting intentions: Mechanisms of retrieval sensitization in prospective memory. In M. Brandimonte, G. O. Einstein, \& M. A. McDaniel (Eds.), Prospective memory: Theory and applications (pp. 93-113). Mahwah, NJ: Erlbaum.

Marsh, R. L., Hicks, J. L., \& Bink, M. L. (1998). Activation of completed, uncompleted, and partially completed intentions.
Journal of Experimental Psychology: Learning, Memory, and Cognition, 24, 350-361. doi:10.1037/0278-7393.24.2.350

Marsh, R. L., Hicks, J. L., \& Bryan, E. S. (1999). The activation of unrelated and canceled intentions. Memory \& Cognition, 27, 320327. doi:10.3758/BF03211415

Marsh, R. L., Hicks, J. L., \& Cook, G. I. (2006). Task interference from prospective memories covaries with contextual associations of fulfilling them. Memory \& Cognition, 34, 1037-1045. doi:10.3758/BF03193250

Marsh, R. L., Hicks, J. L., \& Cook, G. I. (2008). On beginning to understand the role of context in prospective memory. In $\mathrm{M}$. Kliegel, M. A. McDaniel, \& G. O. Einstein (Eds.), Prospective memory: Cognitive, neuroscience, developmental, and applied perspectives (pp. 77-100). New York, NY: Erlbaum.

McDaniel, M. A., Guynn, M. J., Einstein, G. O., \& Breneiser, J. (2004). Cuefocused and reflexive-associative processes in prospective memory retrieval. Journal of Experimental Psychology: Learning, Memory, and Cognition, 30, 605-614. doi:10.1037/0278-7393.30.3.605

Meacham, J. A. (1988). Interpersonal relations and prospective remembering. In M. M. Gruneberg, P. E. Morris, \& R. N. Sykes (Eds.), Practical aspects of memory: Current research and issues. Vol. 1: Memory in everyday life (pp. 354-359). Oxford, UK: Wiley.

Penningroth, S. L. (2011). When does the intention-superiority effect occur? Activation patterns before and after task completion, and moderating variables. Journal of Cognitive Psychology, 23, 140156. doi: $10.1080 / 20445911.2011 .474195$

Schult, J. C. (2011). Zur Verfügbarkeit intentionsrelevanter Informationen in Abhängigkeit von Relevanz und zeitlicher Nähe der zu erledigenden Aufgabenstellung. Unpublished dissertation. Jena, Germany: Friedrich-Schiller-Universität.

Schult, J. C., \& Steffens, M. C. (2011). On the representation of intentions: Do personally relevant consequences determine activation? Memory \& Cognition, 39, 1487-1495. doi:10.3758/s13421-011-0110-3

Smith, R. E. (2003). The cost of remembering to remember in eventbased prospective memory: Investigating the capacity demands of delayed intention performance. Journal of Experimental Psychology: Learning, Memory, and Cognition, 29, 347-361. doi:10.1037/0278-7393.29.3.347

Smith, R. E. (2008). Connecting the past and the future: Attention, memory, and delayed intentions. In M. Kliegel, M. A. McDaniel, \& G. O. Einstein (Eds.), Prospective memory: Cognitive, neuroscience, developmental, and applied perspectives (pp. 29-52). New York, NY: Erlbaum.

Walser, M., Fischer, R., \& Goschke, T. (2012). The failure of deactivating intentions: Aftereffects of completed intentions in the repeated prospective memory cue paradigm. Journal of Experimental Psychology: Learning, Memory, and Cognition, 38, 1030-1044. doi:10.1037/a0027000 